\title{
A GENERAL BOUND FOR OSCILLATORY INTEGRALS WITH A POLYNOMIAL PHASE OF DEGREE $k$
}

\author{
O. Robert and P. SARgos
}

\begin{abstract}
Let $f \in \mathbb{R}\left[X_{1}, \ldots, X_{n}\right]$ be a polynomial of degree $k \geqslant 2$. We consider the oscillatory integral $I(\lambda)=\int \varphi(\mathbf{x}) \mathrm{e}^{i \lambda f(\mathbf{x})} \mathrm{d} \mathbf{x}$, where $\varphi$ is a $C^{1}$ function with compact support. A classical result due to E.M. Stein implies that $I(\lambda)=O\left(\lambda^{-1 / k}\right)$, as $\lambda \rightarrow+\infty$. The exponent $1 / k$ is best possible, as shown by the example $f(\mathbf{x})=f\left(\mathbf{x}_{0}\right) \pm L\left(\mathbf{x}-\mathbf{x}_{0}\right)^{k}$, where $\mathbf{x}_{0}$ is any point in $\mathbb{R}^{n}$ and $L$ is any nonzero linear form on $\mathbb{R}^{n}$. In this paper, we show that, if $f$ is precisely not of the above form, then the stronger bound $I(\lambda)=$ $O\left(\lambda^{-1 /(k-1)}\right)$ holds, and the exponent $-1 /(k-1)$ is best possible.
\end{abstract}

\section{Statement of the result}

We consider a polynomial $f \in \mathbb{R}\left[X_{1}, \ldots, X_{n}\right]$ of total degree $k$, i.e. $f(\mathbf{x})=\sum_{\alpha} a_{\alpha} \mathbf{x}^{\alpha}$, with $\alpha=\left(\alpha_{1}, \ldots, \alpha_{n}\right)$, such that $|\alpha|=\alpha_{1}+\ldots+\alpha_{n} \leqslant k$, for each $\alpha$ in the summation, and such that there exists at least one $\alpha$ with $|\alpha|=k$ and $a_{\alpha} \neq 0$; here, as usual, we have set $\mathbf{x}^{\alpha}=x_{1}^{\alpha_{1}} \ldots x_{n}^{\alpha_{n}}$. Let us denote by $C_{c}^{1}(\Omega)$ the set of all functions $\varphi$ which are $C^{1}$ with compact support contained in the open set $\Omega$. Let $\Delta$ denote the largest size of those $\left|a_{\alpha}\right|$ for which $|\alpha|=k$. Then for any $\varphi \in C_{c}^{1}\left(\mathbb{R}^{n}\right)$, the general bound of Stein's Lemma (cf Lemma 1 below) applies here in the following form :

$$
\left|\int \varphi(\mathbf{x}) \mathrm{e}^{i \lambda f(\mathbf{x})} \mathrm{d} \mathbf{x}\right| \leqslant C_{1}(k)(\Delta \lambda)^{-1 / k}\left(\|\varphi\|_{L^{\infty}}+\left\|\varphi^{\prime}\right\|_{L^{1}}\right) \text {, for all } \lambda>0
$$

where $C_{1}(k)$ is a positive constant that depends only on the degree $k$. Such a bound is quite uniform. Our aim is to improve the exponent $-1 / k$ in $-1 /(k-1)$, providing that $f$ cannot be written as

$$
f(\mathbf{x})=f\left(\mathbf{x}_{0}\right) \pm L\left(\mathbf{x}-\mathbf{x}_{0}\right)^{k},
$$

for some $\mathbf{x}_{0} \in \mathbb{R}^{n}$ and some linear form $L$ on $\mathbb{R}^{n}$. If so it is, we shall say that $f$ satisfies the hypothesis $H_{k, n}$. Our analog of (1.1) is as follows.

Theorem 1. We suppose that $f$ satisfies the hypothesis $H_{k, n}$ and that $\Omega$ is a bounded open set in $\mathbb{R}^{n}$. We then have, for any $\varphi \in C_{c}^{1}(\Omega)$,

$$
\left|\int \varphi(\mathbf{x}) \mathrm{e}^{i \lambda f(\mathbf{x})} \mathrm{d} \mathbf{x}\right| \leqslant C_{2}(f, \Omega)\|\varphi\|_{1} \lambda^{-1 /(k-1)} \text {, for all } \lambda>0
$$

where $C_{2}(f, \Omega)$ is a positive constant which depends only on $f$ and $\Omega$, and where we have set

$$
\|\varphi\|_{1}=\|\varphi\|_{L^{\infty}}+\left\|\varphi^{\prime}\right\|_{L^{\infty}} .
$$

Received by the editors January 12, 2005. 
This bound is far from being as uniform as (1.1), the constant $C_{2}(f, \Omega)$ depending on an abstract partition of unity. However, the exponent $-1 /(k-1)$ is best possible, as shown by the example $f(x, y)=x^{k-1} y$, where in that case the true order of the oscillatory integral (restricted to a neighbourhood of the origin) is given by the stationnary phase method, see e.g. Theorem 3 in $\S 8.3$, CH.II of [1].

Now, we present a slight generalisation of Theorem 1, which may be of some interest, and which does not increase our proof. We consider a Lebesgue-measurable subset $\Gamma$ of $\mathbb{R}^{n}$ which has the following property :

"For any line $D$, the set $D \cap \Gamma$ is the union of at most $N$ segments".

The example we have in mind is the following : $\Gamma$ is the intersection of a compact convex subset of $\mathbb{R}^{n}$ with the set

$$
\left\{\mathbf{x} \in \mathbb{R}^{n} ; Q(\mathbf{x}) \geqslant 0\right\} \text {, where } Q \in \mathbb{R}\left[X_{1}, \ldots, X_{n}\right] \text { has degree at most } N .
$$

Theorem 2. We suppose that all hypotheses of Theorem 1 are satisfied and that $\Gamma$ satisfies (1.4). We then have, for any $\varphi \in C_{c}^{1}(\Omega)$ :

$$
\left|\int_{\Gamma} \varphi(\mathbf{x}) \mathrm{e}^{i \lambda f(\mathbf{x})} \mathrm{d} \mathbf{x}\right| \leqslant N C_{3}(f, \Omega)\|\varphi\|_{1} \lambda^{-1 /(k-1)} \text {, for all } \lambda>0
$$

where $C_{3}(f, \Omega)$ is a positive constant which depends at most on $f$ and $\Omega$.

Of course, Theorem 2 contains Theorem 1 and the rest of this paper is devoted to its proof.

\section{Basic lemmas}

We first recall Stein's fundamental lemma (cf [2], Proposition 5, page 342), with a slight modification concerning the domain $\Gamma$ of integration.

Lemma 1. Let $\Omega$ be a bounded open set in $\mathbb{R}^{n}$ and let $g: \Omega \rightarrow \mathbb{R}$ be a regular function such that the derivative $\partial^{\alpha} g=\frac{\partial^{k} g}{\partial x_{1}^{\alpha_{1}} \ldots \partial x_{n}^{\alpha_{n}}}$, with $k=|\alpha|$, and $k \geqslant 1$, satisfies

$$
\left|\partial^{\alpha} g(\mathbf{x})\right| \geqslant 1, \text { for all } \mathbf{x} \in \Omega
$$

and let $\Gamma$ satisfy (1.4). Then, for any $\varphi \in C_{c}^{1}(\Omega)$, one has

$$
\left|\int_{\Gamma} \varphi(\mathbf{x}) \mathrm{e}^{i \lambda g(\mathbf{x})} \mathrm{d} \mathbf{x}\right| \leqslant N C_{4}\left(\|g\|_{k+1}, k\right)\left(\|\varphi\|_{L^{\infty}}+\left\|\varphi^{\prime}\right\|_{L^{1}}\right) \lambda^{-1 / k} \text { for } \lambda>0
$$

where $C_{4}\left(\|\varphi\|_{k+1}, k\right)$ is a positive constant which depends at most on $k$ and on the maximal size of the derivatives of order $k+1$ of $g$.

Proof. In the case $N=1$, this lemma is exactly Stein's Lemma. For proving it in the case $N>1$, note that Stein's proof uses a linear change of variables which does not alter the property (1.4) and which reduces the problem to a one dimensional oscillatory integral, but, in our case, with several (at most $N$ ) intervals. It is thus obvious that the case $N>1$ reduces to the case $N=1$ by multiplying the final bound by $N$.

The following elementary one dimensional lemma is also needed. 
Lemma 2. Let $k \geqslant 2$ be an integer, $s \geqslant 1$ be real, $\chi:[a, b] \rightarrow \mathbb{C}$ be a $C^{1}$ function, with $0 \leqslant a<b \leqslant 1$. We then have the bound

$$
\int_{a}^{b} \chi(t) \mathrm{e}^{i \lambda t^{k}} t^{s} \mathrm{~d} t=O\left(\|\chi\|_{1} \lambda^{-1 /(k-1)}\right)
$$

where the implied constant depends only on $k$.

Proof. We make a change of variable by setting $t=\tau^{(k-1) / k}$, and we get

$$
\int_{a}^{b} \chi(t) \mathrm{e}^{i \lambda t^{k}} t^{s} \mathrm{~d} t=\int_{a_{1}}^{b_{1}} \chi_{1}(\tau) \mathrm{e}^{i \lambda \tau^{k-1}} \mathrm{~d} \tau
$$

where we have introduced obvious notations. As we have assumed $s \geqslant 1$ and $k \geqslant 2$, we have $\left\|\chi_{1}\right\|_{L^{1}}=O\left(\|\chi\|_{1}\right)$ and $\left\|\chi_{1}\right\|_{L^{\infty}}=O\left(\|\chi\|_{1}\right)$, so that we may apply the Corollary of Proposition 2 in page 332 of [2].

We need also an immediate algebraic lemma.

Lemma 3. Let $P \in \mathbb{R}\left[X_{1}, \ldots, X_{n}\right]$, with $n \geqslant 2$, be a homogeneous polynomial which satisfies

$$
P\left(x_{1}, \ldots, x_{n-1}, 1\right)=0 \text {, for all real numbers } x_{1}, \ldots, x_{n-1} .
$$

Then $P(\mathbf{x})=0$ for all $\mathbf{x} \in \mathbb{R}^{n}$.

Proof. Of course, applying Taylor's formula over the $x_{n}$ variable, we see that $P$ is divisible by $x_{n}-1$.

\section{The local form of the theorem}

We establish now the main intermediate result in the proof of Theorem 2 ; we show how to divide the domain of integration according to the local properties of $f$, considering here the worst case.

Theorem 3. Let $P \in \mathbb{R}\left[X_{1}, \ldots, X_{n}\right]$ be a homogeneous polynomial of degree $k \geqslant 2$, satisfying the property

$$
\text { " There does not exist a linear form } L \text { on } \mathbb{R}^{n} \text { such that } P(x)= \pm L(x)^{k} \text {." }
$$

Then there exists an open neighbourhood $V$ of 0 in $\mathbb{R}^{n}$ such that, for any set $\Gamma$ satisfying (1.4) and any $\psi \in C_{c}^{1}(V)$, one has

$$
\left|\int_{\Gamma} \psi(\mathbf{x}) \mathrm{e}^{i \lambda P(\mathbf{x})} \mathrm{d} \mathbf{x}\right| \leqslant N C_{5}(P)\|\psi\|_{1} \lambda^{-1 /(k-1)},
$$

where $C_{5}(P)$ is a positive constant which depends at most on $P$.

Proof. We divide the proof in several steps.

1) Splitting the domain of integration

We fix a $C^{1}$ test function $\psi$ whose support is contained in $[-1,1]^{n}$. From now on, we shall use the symbol $u \ll v$ to mean that there exists a constant $C$ (which depends at most on $P$ and on other parameters that will be recalled when necessary), such that one has $|u| \leqslant C v$. We then set

$$
I(\lambda)=\int_{\Gamma} \psi(\mathbf{x}) \mathrm{e}^{i \lambda P(\mathbf{x})} \mathrm{d} \mathbf{x}
$$


and we have to prove that

$$
I(\lambda) \ll N\|\psi\|_{1} \lambda^{-1 /(k-1)}, \text { for all } \lambda>0,
$$

providing that $\psi$ has its support contained in a sufficiently small neighbourhood of 0 . We split the domain of integration into $2^{n}$ parts, writing, for each $\varepsilon=\left(\varepsilon_{1}, \ldots, \varepsilon_{n}\right) \in$ $\{-1,1\}^{n}$,

$$
\Gamma_{\varepsilon}=\left\{\mathbf{x} \in \Gamma ; \varepsilon_{j} x_{j} \geqslant 0, j=1, \ldots, n\right\}
$$

so that we have $\Gamma=\cup_{\varepsilon} \Gamma_{\varepsilon}$. Moreover, for each $\varepsilon$, we split $\Gamma_{\varepsilon}$ into $n$ parts : for each $r=1,2, \ldots, n$, we define

$$
\Gamma_{\varepsilon, r}=\left\{\mathbf{x} \in \Gamma_{\varepsilon} ;\left|x_{j}\right| \leqslant\left|x_{r}\right| \text { for } j=1, \ldots, n\right\}
$$

so that we have

$$
|I(\lambda)| \leqslant n 2^{n} \max _{\varepsilon, r}\left|\int_{\Gamma_{\varepsilon, r}} \psi(\mathbf{x}) \mathrm{e}^{i \lambda P(\mathbf{x})} \mathrm{d} \mathbf{x}\right| .
$$

We are going to bound, for instance,

$$
I_{0}(\lambda)=\int_{\Gamma_{0}} \psi(\mathbf{x}) \mathrm{e}^{i \lambda P(\mathbf{x})} \mathrm{d} \mathbf{x}, \text { with } \Gamma_{0}=\left\{\mathbf{x} \in \Gamma ; 0 \leqslant x_{j} \leqslant x_{n}, j=1,2, \ldots, n-1\right\}
$$

and we have to prove that there exists a neighbourhood $V$ of 0 in $\mathbb{R}^{n}$ such that the bound

$$
I_{0}(\lambda) \ll N\|\psi\|_{1} \lambda^{-1 /(k-1)}, \text { for all } \lambda>0 \text { and for all } \psi \in C_{c}^{1}(V)
$$

holds, the implied constant depending at most on $P$ (and thus on $n$ and $k$ ).

\section{2) A change of variables}

We want to prove Theorem 3 by induction on $n$. We note that, for $n=1$, there is nothing to prove, because a homogeneous polynomial of degree $k$ in one variable cannot satisfy (3.1). Thus, we suppose $n \geqslant 2$. If $n \geqslant 3$, we assume that the theorem have been proved up to the dimension $n-1$, and if $n=2$, we have nothing to assume, Lemma 1 being a sufficient reference. In order to make a change of variables which will reduce the dimension (in some way, at least), we define the sets $S=\left\{\mathbf{x} \in[0,1]^{n} ; x_{j} \leqslant x_{n}\right.$ for $\left.j=1, \ldots, n-1\right\}$ and $T=[0,1]^{n-1}$ We set $x_{1}=t u_{1}, \ldots, x_{n-1}=t u_{n-1}, x_{n}=t$, so that we have the general formula

$$
\int_{S} \chi(\mathbf{x}) \mathrm{d} \mathbf{x}=\int_{0}^{1} \int_{T} \chi(t \mathbf{u}, t) t^{n-1} \mathrm{~d} t \mathrm{~d} \mathbf{u}
$$

for any integrable function $\chi$. Now, we define $\gamma(\mathbf{x})$ as being the characteristic function of $\Gamma$, so that we may write

$$
I_{0}(\lambda)=\int_{0}^{1} \int_{T} \gamma(t \mathbf{u}, t) \psi(t \mathbf{u}, t) \mathrm{e}^{i t^{k} \lambda Q(\mathbf{u})} t^{n-1} \mathrm{~d} t \mathrm{~d} \mathbf{u}
$$

where we have set $Q(\mathbf{u})=P(\mathbf{u}, 1)$.

3) An intermediate property

Let $\mathbf{a} \in T$ be fixed. We want to ensure the existence of a neighbourhood $V(\mathbf{a})$ of a in $\mathbb{R}^{n-1}$ such that

$$
\int_{0}^{1} \int_{T \cap V(\mathbf{a})} \gamma(t \mathbf{u}, t) \psi(t \mathbf{u}, t) \mathrm{e}^{i t^{k} \lambda Q(\mathbf{u})} t^{n-1} \mathrm{~d} t \mathrm{~d} \mathbf{u} \ll N\|\psi\|_{1} \lambda^{-1 /(k-1)} .
$$


Suppose first that $Q(\mathbf{a}) \neq 0$, say $|Q(\mathbf{a})|=2 \delta$, with $\delta>0$. Then we choose $V(\mathbf{a})$ so small that $|Q(\mathbf{u})| \geqslant \delta$ throughout $V(\mathbf{a})$. For each fixed $\mathbf{u} \in V(\mathbf{a})$, we bound the integral $\int_{0}^{1} \gamma(t \mathbf{u}, t) \psi(t \mathbf{u}, t) \exp \left(i \lambda Q(\mathbf{u}) t^{k}\right) \mathrm{d} t$ by means of Lemma 2 ; for this, we have to recall that we have fixed $n \geqslant 2$, and to note that the function $t \rightarrow \gamma(t \mathbf{u}, t)$ is the characteristic function of a union of at most $N$ intervals. Integrating then over $\mathbf{u}$, we obtain (3.8) in the case $Q(\mathbf{a}) \neq 0$.

4) We consider now the more difficult case where $Q(\mathbf{a})=0$. We set $\mathbf{u}=\mathbf{a}+\mathbf{v}$ and $R(\mathbf{v})=Q(\mathbf{a}+\mathbf{v}) ; R$ is thus a polynomial in $n-1$ variables, of degree $\leqslant k$, with $R(\mathbf{0})=0$.

We write $R(\mathbf{v})=\sum_{\alpha} b_{\alpha} \mathbf{v}^{\alpha}$, with $\alpha=\left(\alpha_{1}, \ldots, \alpha_{n-1}\right) \in \mathbb{N}^{n-1},|\alpha| \leqslant k$. We dismiss the case $R(\mathbf{v}) \equiv 0$; indeed, this would mean that $P\left(x_{1}-a_{1}, \ldots, x_{n-1}-a_{n-1}, 1\right) \equiv 0$, which is impossible by Lemma 3 .

Thus, we know that there is at least one index $\alpha \neq \mathbf{0}$ such that $b_{\alpha} \neq 0$. We first consider the case where this $\alpha$ satisfies $|\alpha|=l$, with $1 \leqslant l \leqslant k-1$.

For each fixed $t \in[0,1]$, we note that the function $\mathbf{u} \rightarrow \gamma(t \mathbf{u}, t)$ is the characteristic function of a set in $\mathbb{R}^{n-1}$ which satisfies (1.4).

Now, the derivative $\partial^{\alpha} R(\mathbf{v})$ is equal to the constant term $\left(\alpha_{1} !\right) \ldots\left(\alpha_{n-1} !\right) b_{\alpha}$ plus non constant monomials that will be small if we restrict $\mathbf{v}$ to a sufficiently small neighbourhood of $\mathbf{0}$ in $\mathbb{R}^{n-1}$. We have shown that there exists a neighbourhood $W$ of $\mathbf{0}$ in $\mathbb{R}^{n-1}$ and a real $\delta>0$, both depending only on a and $P$ (and, in particular, not on $t$ ) such that $\left|\partial^{\alpha} R(\mathbf{v})\right| \geqslant \delta$ throughout $W$.

We set $V(\mathbf{a})=\mathbf{a}+W$, so that we have $\left|\partial^{\alpha} Q(\mathbf{u})\right| \geqslant \delta$ throughout $V(\mathbf{a})$, and we apply Lemma 1 :

$$
\int_{T \cap V(\mathbf{a})} \gamma(t \mathbf{u}, t) \psi(t \mathbf{u}, t) \exp \left(i \lambda t^{k} Q(\mathbf{u})\right) \mathrm{d} \mathbf{u} \ll N\|\psi\|_{1}\left(\delta t^{k} \lambda\right)^{-1 / l},
$$

for all $\lambda>0$ and each $t \in[0,1]$.

Integrating this inequality over $t$, we set $A(t)=t^{n-1} \min \left\{1,\left(t^{k} \lambda\right)^{-1 / l}\right\}$, and we write

$$
\begin{aligned}
\int_{0}^{1} A(t) \mathrm{d} t & =\int_{0}^{\tau} A(t) \mathrm{d} t+\int_{\tau}^{1} A(t) \mathrm{d} t \\
& \leqslant \int_{0}^{\tau} t^{n-1} d t+\lambda^{-1 / l} \int_{\tau}^{1} t^{n-1-k / l} d t \\
& \ll \tau^{n}\left(1+\lambda^{-1 / l} \tau^{-k / l}\right)+\lambda^{-1 / l}
\end{aligned}
$$

In this last bound, we take $\tau=\lambda^{-1 / k}$ (assuming $\lambda \geqslant 1$, otherwise there is nothing to prove), and we get

$$
\int_{0}^{1} A(t) \mathrm{d} t \ll \lambda^{-n / k}+\lambda^{-1 / l}
$$

From this, we recover (3.8).

5) For proving (3.8), it remains to consider the case where $Q(\mathbf{a})=0$ and where $R(\mathbf{v})$ is a homogeneous polynomial of degree $k$.

But such a situation cannot occur in the case $n=2$. Indeed, $R(v)$ is a homogeneous polynomial of degree $k$ and can be written as $R(v)=b v^{k}$, and thus, $P(x, 1)=$ 
$b(x-a)^{k}$. By Lemma 3 , the only polynomial $P(x, y)$, homogeneous of degree $k$, which satisfies $P(x, 1)=b(x-a)^{k}$ is $P(x, y)=b(x-a y)^{k}$, so that (3.1) is not satisfied.

Now, we suppose $n \geqslant 3$. In the same way as above, we show that $R(\mathbf{v})$ cannot be written as $R(\mathbf{v})= \pm L(\mathbf{v})^{k}$ : otherwise we should have

$$
P\left(x_{1}, \ldots, x_{n-1}, 1\right)= \pm L\left(x_{1}-a_{1}, \ldots, x_{n-1}-a_{n-1}\right)^{k}
$$

and this would imply that

$$
P\left(x_{1}, \ldots, x_{n-1}, x_{n}\right)= \pm L\left(x_{1}-a_{1} x_{n}, \ldots, x_{n-1}-a_{n-1} x_{n}\right)^{k}+P_{0}(\mathbf{x})
$$

where $P_{0}(\mathbf{x})$ is a homogeneous polynomial which satisfies $P_{0}\left(x_{1}, \ldots, x_{n-1}, 1\right)=0$, for all $x_{1}, \ldots, x_{n-1}$. By Lemma 3 , this is possible only if $P_{0}(\mathbf{x}) \equiv 0$. Thus $R$ satisfies (3.1) in the lower dimension $n-1$.

From our recurrence hypothesis (that the theorem is true in the $n-1$ dimensional case), there exists a neighbourhood $W$ of $\mathbf{0}$ in $\mathbb{R}^{n-1}$ such that, setting $V(\mathbf{a})=\mathbf{a}+W$, we have

$$
\int_{T \cap V(\mathbf{a})} \gamma(t \mathbf{u}, t) \psi(t \mathbf{u}, t) \mathrm{e}^{i \lambda t^{k} Q(\mathbf{u})} \mathrm{d} \mathbf{u} \ll N\|\psi\|_{1}\left(t^{k} \lambda\right)^{-1 /(k-1)} .
$$

We integrate this inequality over $t$ as previously (see the corresponding proof in step 4 ), and we recover (3.8).

We have finally proved (3.8) unconditionally when $n=2$, and also for $n>2$, providing that the theorem is true in dimension $n-1$.

\section{6) Conclusion}

We treat together the cases $n=2$ and $n>2$ because they are identical, but one should have to prove firstly the case $n=2$, and then, the case $n>2$ by induction on $n$.

We have to prove (3.5). For each $\mathbf{a} \in T$, we choose a neighbourhood $V(\mathbf{a})$ as in (3.8). Let $\chi_{1}, \ldots, \chi_{s}$ be $C^{1}$ functions on $\mathbb{R}^{n-1}$, each one having his support included in one of the $V(\mathbf{a})$, and such that $\sum_{r=1}^{s} \chi(\mathbf{u})=1$ for all $\mathbf{u} \in T$. We have

$$
I_{0}(\lambda)=\sum_{r=1}^{s} \int_{0}^{1} \int_{T} \gamma(t \mathbf{u}, t) \chi_{r}(\mathbf{u}) \psi(t \mathbf{u}, t) \mathrm{e}^{i t^{k} \lambda Q(\mathbf{u})} t^{n-1} \mathrm{~d} t \mathrm{~d} \mathbf{u} .
$$

We bound each integral in the sum using (3.8) and we obtain (3.5). The proof is complete.

\section{Proof of Theorem 2}

Let $f, \varphi, \Omega$ and $\Gamma$ be as in Theorem 2. For each $\mathbf{x}_{0}$ in the compact $\bar{\Omega}$, we are going to construct a neighbourhood $V\left(\mathbf{x}_{0}\right)$ of $\mathbf{x}_{0}$ in $\mathbb{R}^{n}$, so that, for each $\chi \in C_{c}^{1}\left(V\left(\mathbf{x}_{0}\right)\right)$ and each $\lambda>0$, we have

$$
\int_{\Gamma} \chi(\mathbf{x}) \mathrm{e}^{i \lambda f(\mathbf{x})} \mathrm{d} \mathbf{x} \ll N\|\chi\|_{1} \lambda^{-1 /(k-1)},
$$

where the implied constant depends at most on $f$. Assuming (4.1), it is easy to deduce (1.5) with a partition of unity, in the same way as above. Now, our aim is to prove (4.1). 
We fix $\mathbf{x}_{0} \in \bar{\Omega}$. We set $P(\mathbf{y})=f\left(\mathbf{y}+\mathbf{x}_{0}\right)-f\left(\mathbf{x}_{0}\right) ; P$ is a polynomial of degree $k$, which is not of the form $\pm L(\mathbf{y})^{k}$. We have to find a neighbourhood $W$ of $\mathbf{0}$ in $\mathbb{R}^{n}$ such that we have

$$
\int_{\widetilde{\Gamma}} \chi(\mathbf{y}) \mathrm{e}^{i \lambda P(\mathbf{y})} \mathrm{d} \mathbf{y} \ll N\|\chi\|_{1} \lambda^{-1 /(k-1)}, \text { for } \chi \in C_{c}^{1}(W) \text { and } \lambda>0,
$$

where we have set $\widetilde{\Gamma}=-\mathbf{x}_{0}+\Gamma$, and where the implied constant depends at most on $f$.

As $P$ vanishes at $\mathbf{0}$, either $P$ is a homogeneous polynomial of degree $k$, or we have

$$
P(\mathbf{y})=\sum_{\alpha} a_{\alpha} \mathbf{y}^{\alpha}, \text { where } a_{\alpha} \neq 0 \text { for some } \alpha \text { with } 1 \leqslant|\alpha| \leqslant k-1 .
$$

In the first case, which is the more difficult, (4.2) is precisely the conclusion of Theorem 3, so that we may suppose that (4.3) holds. Let $\alpha \in \mathbb{N}^{n}$ such that $1 \leqslant$ $|\alpha| \leqslant k-1$, and $\left|a_{\alpha}\right|=2 \delta$ for some $\delta>0$. Then there exists a sufficiently small neighbourhood $W$ of $\mathbf{0}$ in $\mathbb{R}^{n}$ such that the derivative $\partial^{\alpha} P$ satisfies $\left|\partial^{\alpha} P(\mathbf{y})\right| \geqslant \delta$ throughout $W$.

Thus we may apply Lemma 1 and this implies precisely (4.2). The proof of Theorem 2 is now complete.

\section{References}

[1] V. Arnold, A. Varchenko, S. Goussein-Zade, Singularités des applications différentiables, Vol. II. (Russian) "Nauka", Moscow, 1984. Translation: Birkhuser Boston, Inc., Boston, MA (1988).

[2] E.M. Stein, Harmonic analysis: real-variable methods, orthogonality, and oscillatory integrals, Princeton Mathematical Series, 43, Princeton University Press, Princeton, NJ, 1993.

Institut Elie Cartan, Université Henri Poincaré, Nancy 1 B.P. 239, 54506 VANDOEUVRE-LES-NANCY, FrANCE

E-mail address: robert@iecn.u-nancy.fr, sargos@iecn.u-nancy.fr 